\title{
Molecular, Sequencing and Bioinformatics of Insulin- like growth factor 1(IGF-1) gene and Transforming Growth Factor $\beta 2$ gene Associations with growth traits in three strains of Chicken
}

\section{Waleed S. El-Tahawy ${ }^{1}$ and Abdel-Rahman M.M. ${ }^{2}$}

1. Animal and Poultry Production Department, Agriculture Faculty, Damanhour University

2.Genetic Engineering and Biotechnology Laboratory, Plant Pathology Department (Genetic Branch), Agriculture Faculty, Damanhour University

Corresponding author. E- mail address : mm.rahman@agr.dmu.edu.eg

Abstract

Molecular analysis is an easier means to identify and isolate a specific gene which has imperative function for growth, body composition, fat deposition, metabolic and skeletal traits as well as the molecular genetics selection on individual genes is a very efficient method to genetically improve economically important traits in chickens. Insulin- like growth factor 1 ( IGF-1)is a member of a heterogeneous group of peptides with important growth.Transforming growth factor $\beta$ ( TGF- $\beta$ ) belongs to a large family of growth and differentiation factors that play a pivotal role in a great variety of biological activities including morphogenesis, development and differentiation. DNA was extracted from 48 chickens sampled from three strains Lohman (17), Sinai (24) and Gimmizah (7) IGF-1 gene and TGF $\beta 2$ gene were amplified using PCR protocol. Electrophoresis was carried out on the products of PCR, bands viewed on transilluminator. The size of IGF-1 gene was $675 \mathrm{bp}$ while the size of TGF $\beta 2$ gene was $188 \mathrm{bp}$. Sharp bands were purified and sequenced and used the dendrogram to show the relationships between other vertebrate species

Key words: Chickens, IGF-1 gene , TGF $\beta 2$ gene , DNA extraction, purification , Sequencing and Bioinformatics 


\section{Introduction}

\subsection{The importance of IGF-1 gene in chickens}

Insulin- like growth factor 1 ( IGF-1)is a member of a heterogeneous group of peptides with important growth- promoting effects in vitro as well as in vivo. It plays a fundamental role in postnatal chicken growth as a major mediator through which growth hormone exerts its biological effects.(Sakr et al., 2014)The genetic architecture of quantitative traits may be gained by systematically analyzing genetic markers in major metabolic pathways ( $\mathrm{Li}$ et al., 2008)Insulin- like growth factor I ( IGF-I) and insulin -like growth factor II (IGF-II) constitute specific ligands that belong to a complex system also comprising in mammals specific membrane receptors: IGF type I receptor and mannose 6- phosphate/ IGF-2 receptor and associated binding proteins IGFBP1 to IGFBP6. These molecules are essential for normal growth and development (LeRoith, 1996.) IGF-I is considered as an attractive candidate gene to search for DNA polymorphisms (Lee et al., 1989) The use of molecular marker assisted selection has proven to be efficient and lead to the improvement in production performance in animals (Fang et al.,2008) IGF-I gene in the avian is compact and occupies about $48 \mathrm{~kb}$ of chromosome one. IGF-I comprises four exonsand spanning $210 \mathrm{bp}$ and encodes a single chain polypeptide of 70 amino acids(Amills et al., 2003) IGF regulates the metabolism of glucose, fat and muscle protein during post -hatch development (Buyse and Decuypere, 1999; Conlon and Kita 2002) IGF-I is a candidate gene for growth , body composition and metabolism, skeletal characteristics and growth of adipose tissue and fat deposition in chickens . IGF-I gene may play important roles in growth of multiple tissues, including muscle cells, cartilage, and bone. (Zhou et al., 2005)Insulin- like growth factors ( IGFs)signaling system plays an important role in promoting growth and development in vertebrates (Moriyama et al., 2000; Ohlsson et al., 2009)IGF-I stimulate the proliferation, differentiation, and metabolism of myo-genic cell lines from different species.

\subsection{IGFs regulate body and muscle growth in chicken}

Insulin- like growth factor-1 (IGF-1) consist of a family of polypeptide hormones structurally associated with insulin with multiple metabolic and anabolic functions. The (IGF-1) stimulate the proliferation, differentiation and metabolism of myogenic cell lines from different species (Florini et al., 1996). The (IGF-1) gene may play important roles in growth of multiple tissues, 
including muscle cells, cartilage and bone (Zapf and Froesch, 1996). In chickens divergently selected for high or low growth rates there significantly higher IGF1 mRNA levels in the high growth rate line than in the low growth line (Beccavin et al., 2001). The IGF-1 therefore, was selected as a biological candidate gen to investigate growth, body composition metabolic and skeletal traits in chickens. The chickens IGF-1 gene maps to $165.95 \mathrm{cM}$ on chromosome1.( Sewalem et al., 2002) found $160 \mathrm{cM}$ in a broiler layer F2 population on chromosome1. (Zhou et al., 2005) found the (IGF-1) promoter polymorphism with growth, body composition, skeleton integrity and metabolic traits in broiler chicken. Joromir et al., 2011 detected the relationship between IGF-1 gene polymorphism and growth traits the breast muscle and leg muscle weight were higher in broiler line cobb 500 .

The candidate gene associated with increased meat production is Insulin-like growth factor I (IGF-I) also known as somatomedin is located at chromosome 1: 55,432,530-55,480,957 in the forward strand (Galgal4:CM000093.3) Chromosome 1 - NC_006088.4, the position of IGF-1 on chromosome 1NC_006088.4 from ENSEMBL IGF-1 protein consists of 70 amino acids in a single chain with three intermolecular disulfide bridges. IGF-1 has a molecular weight of 7,649 Daltons (NCBI). IGF-1 10 encodes a protein similar in molecular structure to insulin (Akaboot, Duangjinda, Phasuk, \& Kaenchan, 2012)

\subsection{The importance of TGF 2 in chickens}

Transforming growth factor $\beta$ ( TGF- $\beta$ ) belongs to the TGF $\beta$ super family, and TGF $\beta 1$ (chickTGF $\beta 4$ ), 2 and 3 have been identified in mammals and avians (Kingsley 1994). Transforming growth factor $\beta$ ( TGF- $\beta$ ) belongs to a large family of growth and differentiation factors that play a pivotal role in a great variety of biological activities including morphogenesis, development and differentiation (Burt and Law 1994; Piek et al., 1999; Saxena et al ., 2009).TGF $\beta$ has been demonstrated to regulate many developmental processes. During lung development, TGF $\beta$ is localized in stromal cells and inhibits branching morphogenesis in organ culture (Heine et al., 1990 ; Serra et al., 1994 ) and TGF $\beta$ s has been implicated in cartilage and bone formation (Allistone et al., 2008) TGF $\beta$ activation is controlled by a variety of mechanisms (Dabovic and Rifkin , 2008) Transforming growth factor- betas are multifunctional cytokines that have been implicated in the regulation of a broad range of biological processes, including cell proliferation, cell survival and cell differentiation ( 
Okamura et al., 2015). Transforming growth factor $\beta$ is composed of 4 isoforms: TGF- $\beta 1$, TGF- $\beta 2$, TGF- $\beta 3$ and TGF- $\beta 4$ in chickens (Burt and Paton, 1991;Roberts and Sporn, 2012) Afourth isoform has been described in chickens ( Jakowlew et al., 1988)TGF $\beta$ is a superfamily protein consisting of three isoforms (TGF- $\beta 1$, TGF- $\beta 2$ and TGF- $\beta 3$ ) and responsible for different biological processes like growth, development, tissue homeostasis, adhesion, apoptosis and regulation of the immune system(Kubiczkova et al., 2012; Pardali and TenDijke, 2012)TGF- $\beta 1$, TGF- $\beta 2$, TGF- $\beta 3$ are known in mammals and birds. TGF- $\beta 1$, TGF- $\beta 2$, TGF- $\beta 3$ have high sequence homology, bind to the same receptors and show similar biological functions of the three isoforms. TGF- $\beta 1$ and to a lesser extent TGF- $\beta 2$ play an integral role in maintaining immune tolerance( Okamura et al., 2015). Molecular studies have identified the avian TGF- $\beta 4$ as the ortholog of human TGF- $\beta 1$ ( Halper et al.,2004)

The chicken is the first non- mammalian amniote whose genome was sequenced.The total length of the sequence of the chicken genome is more than a billion nucleotides that encode about 15500 genes (Ruffier et al., 2017)The chicken genome has a relatively low content of repetitive sequences (11\%) compared to mammals. The comparison between the human genome, chicken and fish showed that about one third of all genes are common to all three species. The chicken has become a model bird organism. Sequencing allows for deciphering an enormous amount of genetic information .Improvement in sequencing methods depends to a large extent on the competitiveness between leading producers of innovative sequencers ( Patel and Jain 2012)

\section{$\underline{\text { 2.Materilas and Methods }}$}

The present study was carried out at the poultry research unit (El-Bostan farm) Department of animal and poultry production, faculty of agriculture Damanhour University. The experiment aimed to evaluate the growth production trait of three chicken strains. Lohman brown (LB), Sinai (Si), and Gimmizah (GM ) raised under Egyptian environmental condition Also, to detected two gene IGF1 and TGF $\beta 2$ affected of some growth traits.

\subsection{Sinai strain (SI)}

Sinai chickens were originally obtained from the desert areas of north and went Sinai governments. The Sinai breed probably is originated from the natural cross between some foreign breeds with local chicken heard in Sinai governorates since were 1945

\section{$\underline{\text { 2.2 Gimmizah strain (GM) }}$}


Gimmizah strain is a synthetic local strain chickens which developed at the ministry of agriculture. Gimmizah poultry research station. The formation of Gimmizah strain started in early (Mahmoud et al., 1982) the scheme of formation of Gimmizah strain including two way cross between Dokki 4 x Bren Plymouth rock

\subsection{Lohman brown strain (L.B)}

Lohman brown strain is a commercial strain selected to egg production traits.

\section{$\underline{2.4 \text { Flock management }}$}

At hatching day all chicks were removed from the hatching wing banded and weighed. All chicks were brooded in floor brooding with wood shoving litter. The chicks strains placed in floor brooders at a starting temperature of $33 \mathrm{C}$ during the first 3 days then the brooder temperature was decreased gradually $3 \mathrm{C}$ each week to reach 22-20 C at 56 days of age. At eight weeks of age all chicks were arrowed to hearing house. All chicks were exposed to continuous artificial light for 24 hours during the first weak of age then the light was decreased gradually to reach the natural day light at 8 weeks of age. All birds were housed in the same room and have similar management and environmental condition throughout the whole experimental period. All chicks were fed adlibitum during brooding hearing and growing periods on a diet containing $21 \%$, crude protein and $2900 \mathrm{kcal} \mathrm{ME} / \mathrm{kg}$ diet respectively.. All chicks were vaccinated against diseases and were treated. At 12 weeks of age $3 \mathrm{ml}$ blood sample was collected from wing vein

\subsection{Studied traits, Statistical Analysis and Data collection}

Body weight was recorded for every individual bird to the nearest $0.1 \mathrm{~g}$ from hatch to 12 weeks of age (BW0, BW2, BW4, BW8, andBW12).Daily body weight gain during the different growth periods was studied $(0-2,2-4,4-8,8$ 12and0-12)and calculated as follows: Daily weight gain $=(\mathrm{W} 2-\mathrm{W} 1) /$ INTERVAL (days). Where : W1:weight at the beginning of the period $(\mathrm{g})$ and W2 : weight at the end of the period(g).Interval :the number of days between weighing dates. Data analyses were carried out using PROC GLM in SAS (2004) using the following model:

$$
Y_{i j}=\mu+S_{j}+e_{i j}
$$


Where Yij= observation , $\mu=$ general mean, $\mathrm{Sj}$ : effect of strain and eij: random error.

The data were collected from birds from three strains of (SI) strain, (LB) strain and of (GM) strain. The number of birds were 260 , 156and 162 from SI, LB and GM strain respectively.

\subsection{DNA extraction and primer design}

Chicken genomic DNA was extracted from the blood samples using Norgen Biotek kit then quantified using spectrophotometer (pg T80) and final concentration for PCR reaction were 50-100 ng/ul (Table 1)PCR primers were designed with NCBI and primers

\begin{tabular}{|l|l|l|}
\hline Gene & Primers & $\begin{array}{l}\text { Product } \\
\text { size }\end{array}$ \\
\hline $\begin{array}{l}\text { IGF } \\
\text { NC_006088.5 }\end{array}$ & $\begin{array}{l}\text { F5' CATTGCGCAGGCTCTATCTG 3' } \\
\text { R 5' CGAACTTCCCGAAGAGAACT3' }\end{array}$ & 813bp \\
\hline TGF 2 & F 5' GTGCCTCACCGCTATTTCTG 3' & 188bp \\
NC_006090.4 & R 5' GAAGAGGAGGCTCAGGAGA 3' & \\
\hline
\end{tabular}

\section{Table 1. Primers for IGF-1 and TGFß2 used in this study}

PCR amplification was carried out in 25 ul reaction volumes containing master mix 2x PCR (promega, Madison, USA) primers and DNA.The program for IGF was $95^{\circ} \mathrm{c}$ for $5 \mathrm{~min}$ followed with 35 cycles of denaturation at $95^{\circ} \mathrm{c}$ for 45 $\mathrm{sec}$, annealing at $54.8^{\circ} \mathrm{c}$ for $30 \mathrm{sec}$. and extension at $72^{\circ} \mathrm{c}$ for $45 \mathrm{sec}$ then final extension at $72^{\circ} \mathrm{c}$ at $5.00 \mathrm{~min}$. The program for TGF $\beta 2$ was $95^{\circ} \mathrm{c}$ for $5 \mathrm{~min}$ followed with 35 cycles of denaturation at $95^{\circ} \mathrm{c}$ for $45 \mathrm{sec}$, annealing at $60^{\circ} \mathrm{C}$ for $30 \mathrm{sec}$. and extension at $72{ }^{\circ} \mathrm{c}$ for $30 \mathrm{sec}$ then final extension at $72^{\circ} \mathrm{c}$ for 5.00 min.The amplified PCR products were separated by electrophoresis on $1.5 \%$ agarose in $1 \mathrm{X}$ TAE buffer, ethidium bromide and visualized with uv light of transilluminator (Genetics Nippon)

\subsection{PCR purification}

Positive sharp bands were purified using Gene JET gel extraction kit (thermo scientific) and sequenced directly.

\section{$\underline{2.8 \text { Bioinformatics analysis of sequencer results }}$}


The sequences of the positive bands were analyzed using the Blast search service at NCBI website.The sequences for 4 samples (strain lohman), 3 samples (strain Sinai) and one sample for( strain Gimmizah).The sequencer results were aligned and compared with that of other different representative species using Blastx and Blastn.

\section{$\underline{\text { 3.Results }}$}

\subsection{The body weight for three strains}

The body weight at different age in three strains lohman brown (LB), Gimmizah and Sinai are shown in table (2). The results showed highly significant $(\mathrm{p} \leq 0.01)$ between the three strains. Sinai chickens have lower body weight at hatch and 2 weeks $35.7 \mathrm{gm}$ and $115.23 \mathrm{gm}$ respectively.

\begin{tabular}{|l|l|l|l|l|}
\hline \multirow{2}{*}{ Trait } & \multicolumn{3}{|c|}{ Strain } & \multirow{2}{*}{ ANOVA } \\
\cline { 2 - 4 } & $(\mathrm{GM})$ & \multicolumn{1}{|c|}{ (SI) } & \multicolumn{1}{|c|}{ (LB) } & \\
\hline BW0 & $38.13 \pm 0.29^{\mathrm{A}}$ & $35.73 \pm 0.17^{\mathrm{C}}$ & $36.64 \pm 0.16^{\mathrm{B}}$ & $* *$ \\
BW2 & $131.97 \pm 1.54^{\mathrm{A}}$ & $115.23 \pm 0.84^{\mathrm{C}}$ & $123.16 \pm 0.84^{\mathrm{B}}$ & $* *$ \\
BW4 & $246.77 \pm 4.59^{\mathrm{A}}$ & $218.04 \pm 2.14^{\mathrm{B}}$ & $203.26 \pm 2.32^{\mathrm{C}}$ & $* *$ \\
BW8 & $656.50 \pm 13.70^{\mathrm{A}}$ & $568.1 \pm 6.82^{\mathrm{B}}$ & $505.14 \pm 6.59^{\mathrm{C}}$ & $* *$ \\
BW12 & $972.68 \pm 22.23^{\mathrm{A}}$ & $852.80 \pm 8.19^{\mathrm{B}}$ & $757.18 \pm 7.76^{\mathrm{C}}$ & $* *$ \\
\hline
\end{tabular}

Table (2): Least square means $(X) \pm$ standard error (SE) for body weight at different age in three strains Lohman brown (LB) ,Gimmizah ( GM) and Sinai (SI).Means having different letters in each effect are differ significantly $(\mathrm{P} \leq 0.05)$. A, B between strain ** Significantly at $\mathrm{P}$ $\leq 0.01$.

The body weight of (GM) strain chicken at hatch, 2,4,8 and 12 weeks of age were $(38.13,131.97,246.77,656.5$ and $972.68 \mathrm{gm})$ respectively.The body weight of( LB) strain had lower body weight at 4,8 and 12 weeks of age were (203.26, 505.14 and $757.18 \mathrm{~g}$ ) respectively. The body weight of (GM )strain have highest body weight than LB strain and (SI) strain at different age. The body weight gain at different age in all strain chickens were show in table (3). The results showed highly significant $(\mathrm{p} \leq 0.01)$ between the three strains The highest body weight gain was obtained by GM strain at different age. The LB chicken strain showed lower body weight gain from (2-4,4-8,8-12and 0-12) weeks than the SI and GM strains. However the SI strain have lower body weight gain from 0-2 weeks than GM and LB strains.

\begin{tabular}{|l|l|l|l|l|}
\hline \multirow{2}{*}{ Trait } & \multicolumn{3}{|c|}{ Strain } & \multirow{2}{*}{ ANOVA } \\
\cline { 2 - 4 } & $(\mathrm{GM})$ & \multicolumn{1}{|c|}{$(\mathbf{S I})$} & $(\mathbf{L B})$ & \\
\hline BWG0-2 & $93.84 \pm 1.50^{\mathrm{A}}$ & $79.49 \pm 0.84^{\mathrm{C}}$ & $86.51 \pm 0.82^{\mathrm{B}}$ & $* *$ \\
BWG2-4 & $114.80 \pm 4.38^{\mathrm{A}}$ & $102.81 \pm 1.54^{\mathrm{B}}$ & $80.10 \pm 2.18^{\mathrm{C}}$ & $* *$ \\
BWG4-8 & $409.72 \pm 9.78^{\mathrm{A}}$ & $350.86 \pm 6.17^{\mathrm{B}}$ & $301.87 \pm 6.39^{\mathrm{C}}$ & $* *$ \\
\hline
\end{tabular}




\begin{tabular}{|l|l|l|l|l|}
\hline BWG8-12 & $316.18 \pm 15.52^{\mathrm{A}}$ & $283.89 \pm 5.09^{\mathrm{B}}$ & $252.03 \pm 5.46^{\mathrm{C}}$ & $* *$ \\
BWG0-12 & $934.55 \pm 21.98^{\mathrm{A}}$ & $817.07 \pm 8.11^{\mathrm{B}}$ & $720.54 \pm 7.72^{\mathrm{C}}$ & $* *$ \\
\hline
\end{tabular}

Table (3): Least square means $(X) \pm$ standard error (SE) for body weight gain at different age in three strains Lohman brown (LB), Gimmizah ( GM) and Sinai (SI).Means having different letters in each effect are differ significantly $(\mathrm{P} \leq 0.05)$. A, B between strain **Significantly at $\mathrm{P} \leq$ 0.01 .

\subsection{PCR analysis for IGF}

Polymerase chain reaction (PCR) was used to amplify fragments. The amplified product was $675 \mathrm{bp}$ an indiction that the amplicon was IGF1 For lohman strain 4 bands were appeared in line 12,14,58 and 97.Figure 1.For Sinai strain three bands were appeared in line 8,22 and 26.Figure 2.For Gimmizah only one band was appeared in line 81.Figure 1

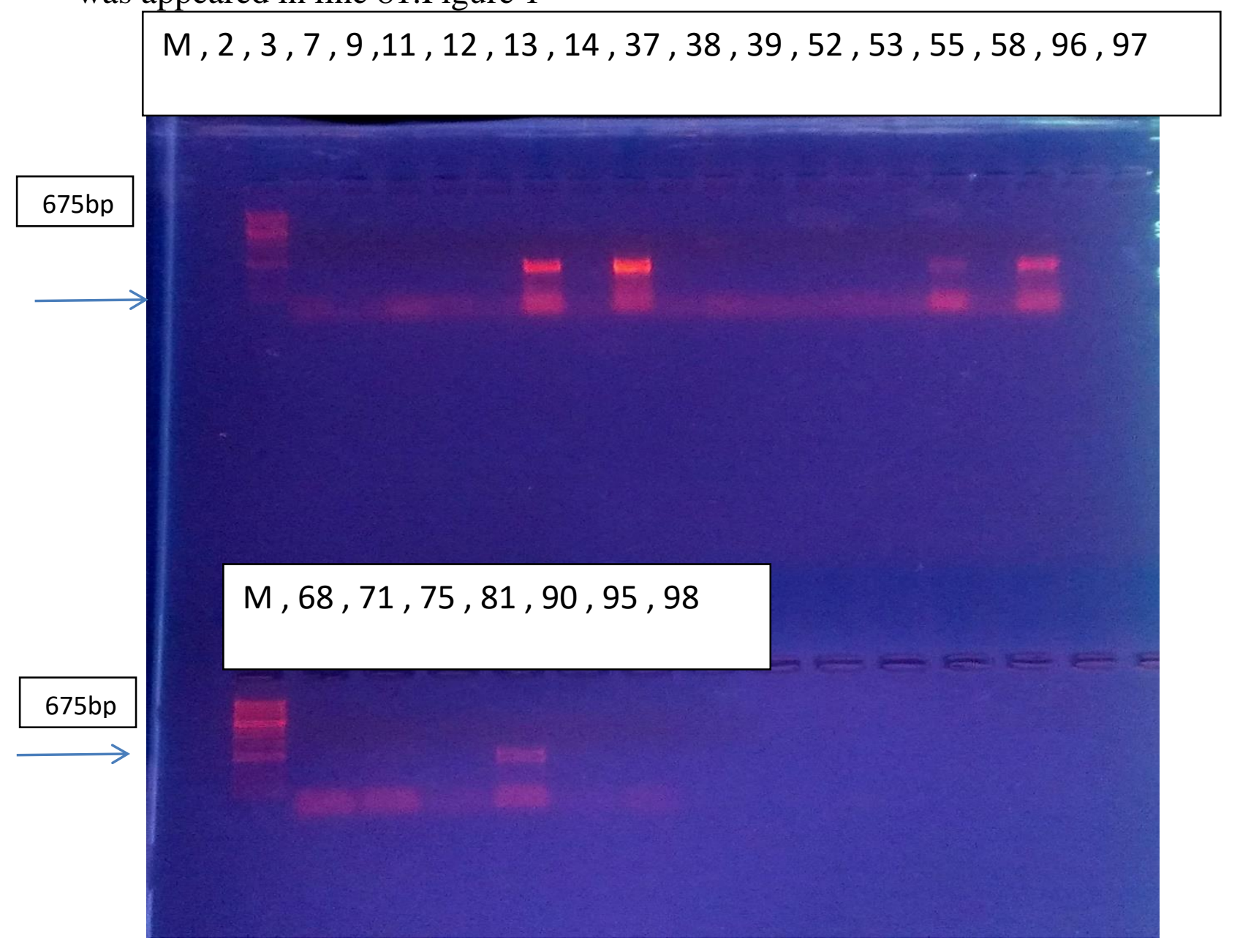

Figure 1. PCR amplification gel image of the insulin - like growth factor 1 gene of chicken (Lohman strain up, Gimmizah strain down, $M=1 \mathrm{~kb}$ ladder) 
Gimmizah strain tree was shown two branches the first was Sphingobium and the second branch was divided to 4 clusters of a- proteobacteria (Fig 19)

\begin{tabular}{|c|c|}
\hline $\begin{array}{l}\text { IGF- } 1 \\
675 \mathrm{bp} \\
\end{array}$ & \\
\hline $\begin{array}{l}\text { Lohman } 12 \\
\text { (F) } \\
\text { Fig } 3\end{array}$ & $\begin{array}{l}\text { NNNGTNNNTTGNCCNNCNACTNCAGGAGNACGTTGGGAACCCGAGNNCGCNGCCNNCTCCCC } \\
\text { AGGNGCNCANGTTCGGGGNGGGNCNTNNGNGGGGGGGAGNGGGGCGAGGATANCGGACTCT } \\
\text { TTTCANCTCGCTGCNGACATCCNACNCNAATAGNNCTTGACCANTAANGACCCGTCTCTCGNG } \\
\text { AGAGTTGCCCTNGNCGCAGNAGNCTACTATCNGCCACCGCG }\end{array}$ \\
\hline $\begin{array}{l}\text { Lohman } 12 \\
\text { (R) } \\
\text { Fig } 4\end{array}$ & $\begin{array}{l}\text { NNNNNTCTNCNANACCNANNATGCNNNNNTCNANGGGAGCNNNNCGCGAGGCTTTCTCGGG } \\
\text { GGGCTGGGCCAATNCGGGGGCCNANANNNNGGGGGTGGGAGGCGNGGCGTGGATCGCAACA } \\
\text { CCTCCGCTCCAGTTCGCTACCCGAGCAGCGACCACACNTGGANCTTGANGNNTTAAGCAGTG } \\
\text { GTCCCCTCGAGAGAGGGTTGCCGATACCCATCCCGGCTACGTTCTGCTACCGCTTNAGGGATC } \\
\text { GCAGTCGCGAAGTAAAGGCCNAANACCCGGCTCGCTCCCGGNTAAAGAGATGTTGCGAGTCG } \\
\text { CTCCGCCCGCCCTACTCCCTCTCATATGGCTTATCCCACTACN }\end{array}$ \\
\hline $\begin{array}{l}\text { Lohman } 14 \\
\text { (F) } \\
\text { Fig } 5\end{array}$ & $\begin{array}{l}\text { NNNNNNGNNNGNTNNNNNNCGNGNNATTACNNCAGANNGGGATTTAGAGAATCCTCCATTT } \\
\text { ATCTACATTACACAGACACTGTAGACAGGAANCAGCTGGGGGAGCATTTGCCTTCTCTCTCTC } \\
\text { TCCCTCTTCTGGCAAAGTTACCGAGTAAGGACTTTTTTGGGCATGGAGACTAATAACATCATA } \\
\text { CCTTTGCATTTTAAAACTAGAGCACATATACATATTTTTTCCCCTTTAAAAGAATAGCTTGACA } \\
\text { GTGATCTGGGTCGCATAATATGTGCCNATGTGGCTGTTCCCTCCAGCNTAAAGATATTCTTCG } \\
\text { ATGGGGTCTGCCGCCATTAATCNCTCTCGATGC }\end{array}$ \\
\hline $\begin{array}{l}\text { Lohman } \\
14(\mathrm{R}) \\
\text { Fig } 6\end{array}$ & $\begin{array}{l}\text { GNNNNNNNNNNNNNTCTAGTACTGTAATTCTAGGAATTGCATGACTTCTGTGTGGTTGCTAAA } \\
\text { GGATCGCAGCGCTGTTTTGCTCTNNTTACAAGCAGTGCTCAACAACTCTTGTACTTTTAGCAA } \\
\text { ACTACCAGCTGCTGCTTTAGGGTTAATAGCACCGAGAGTCACTTGTTTTATTTGTTAGTCATCA } \\
\text { TGTCGGCGTGCCTGCTTTATACCGATCCCCTGTACTTACTGCAACCGCTTGNGGTAGCGCGGT } \\
\text { TCCGTAGGATANGCCGANNNCNNTTCGCTCCCGGNGCTCGAGATTTTNCGATTCGCTCCCNCG } \\
\text { CCATTTCTCCCTCCCNTATGCTTACCCGACTACTTCCGCTCNGTTTCCCAACCGAATTACCNCA } \\
\text { TGTAGGCTAAGTCATCTGCTCACACTCAGCGCGTGTATCACTGACCCCCACTCTNATTGTAGC } \\
\text { TGGTCNNGAGGCTTGCGTCTCGGTCGTACGGCTTNCTANNACTCCGACNNTTCGGANGCCTCA }\end{array}$ \\
\hline $\begin{array}{l}\text { Lohman } 58 \\
\text { (F) } \\
\text { Fig } 7\end{array}$ & $\begin{array}{l}\text { NNNNNNNNNNNNNNNNNNNNNNNNNNNNNNAGACCTTGTGTGGCCAGTGCCNTCNTCTTCG } \\
\text { CTGCTCCTGGNGTANGGGAAGACCGTCCNNTNTTTNANANACGTTTTTTTGACGGAGTCTTCT } \\
\text { GCATTTTGCTGNCTCCCCTGCCNATCTNCAANAAGNGACNCTTNNGCACCCGTNACGTCANGT } \\
\text { GANTTGCCCCATACGCNTCCCCTCTAGTTTTCCNCAGTTTNNTAAACTCANTCNCGTGANCAN } \\
\text { GNCNANTN }\end{array}$ \\
\hline $\begin{array}{l}\text { Lohman } 58 \\
\text { (R) } \\
\text { Fig } 8\end{array}$ & $\begin{array}{l}\text { NNNNNNNNNNNCNCNCNNNCNNCNNANGGAANNTGTGTGGNGTTGTTANCCGCNTCTTCTCC } \\
\text { GGTCNTGTTGANAGGGGGNGNACGGNTNTCCTNNNGGANGGCTTCCTTTTTCGCGGANTNTT } \\
\text { GAGCAACATGGTGCCTCCTGCGACTACNCCCTTANAAGGACCCGTAAANCTCGTGACCTCNN } \\
\text { GAGATNCTGCCCTACACGCGTNGNCNCTAGTTTCTNCCCCACTTTATATATC }\end{array}$ \\
\hline $\begin{array}{l}\text { Lohman } 97 \\
\text { (F) } \\
\text { Fig } 9\end{array}$ & $\begin{array}{l}\text { NNNNNNNNNGNNNNNNNNNNCNCTGCNNNNTCNNGGGNCTCCNAGGCGCGAGGCTTTGTTA } \\
\text { GTGGTCAGCGGCAAGATTCCGGGCTACACCANTACCAANGGTGTGATGAGTGGCGTGGATAC } \\
\text { NCGCACCACGGTTCAGGCTCGCTTCCGGGCCGGCGACGACTATTAGAGCTTGCAGCCTGAAG } \\
\text { CACTGGTCCCCTCGGGTGAGGCTTCCCTATACCCAGCCCCNTTAGTTTCGGCGACNGCTNNNG } \\
\text { GGATCGCAGTCGCGGANTATAGGCCNATGTCNCTGTTCGCCCAGGCGAAAGGTCTTCTTCGCT } \\
\text { GGGCTCTGCGCCATTAATCCCTCTCGTANGCTTTTCCGACTACTTGCACTCNGTTTCCCAACCG } \\
\text { ACTTTCCGCATGTCGGCTATGTCATAGGCCCACACNCAGAGGGTGTNGCACTGATGGACNCTC } \\
\text { TNACTCCAANCTGGTCAAGGGGGTNGCCCGCN }\end{array}$ \\
\hline $\begin{array}{l}\text { Lohman } 97 \\
\text { (R) } \\
\text { Fig } 10\end{array}$ & $\begin{array}{l}\text { NNNNNNNNNNNNCNANGNATGCNGNNTCGNCGNGAGCGCGAGGCGCGAGGCCTTGTCGGTG } \\
\text { TTCTGGGCCAGAGNCGGGCTACGACCATCCCNNNGGTGTGATGACGGGCGTGGAGAGAGGCA } \\
\text { CCTCCTCTCCGGTGCGCTTCACGCGCGGCGACCACTATTGNAGCTTGAACCCTTCAGGTCTGT } \\
\text { TTCCCGCGGGAGAGGCCGCCGGATACGCCGGAGGCATCCTTTCTGCGGCAGCGGACGGGATC } \\
\text { GCGGTTGCAGAANNAGTGCCNAATAGCCTGTTTGNCCCGCGTAAAGGTCTTCTGCGCTGGGG } \\
\text { GCTGCCGCCCNAATCCCTGCAAGATGCTTACCCCACTCCTTGCACTCGGATTGCCGACCGTCC } \\
\text { TTCCGTATGTGACTATGGCACCAGCTCACGGGCAGAGTGGGTGGCGCTGATCCACACNCTGA } \\
\text { GTCTGNCCAGGTCANTTCGCTTGCCCTCGTTCATTCTGCTGCGCGACGCCGCGGACGAATTCG } \\
\text { CGAGCCCAATC }\end{array}$ \\
\hline $\begin{array}{l}\text { Sinai } 8(\mathrm{~F}) \\
\text { Fig } 11\end{array}$ & $\begin{array}{l}\text { GNNNNNNNNGNNNNNCGNGNNNNTAGCAACAGAGAGGGATTAGAGANNTCCTCACATTTAT } \\
\text { CTACATTACACAGACACTGTAGACAGGAANNAGCTGGGGGAGCATTTGCCTTCTCTCTCTCTC } \\
\text { CCTCTTCTGGCAAAGTTACCGAGTAAGGACTTTTTTGGGCATGGTGACCATTAACATCATATC } \\
\text { CTTGCNTTTTAAAACTAGAGGACACCAGAATCTTTTTTTTCTGCTTCAATGAACGGGACGAAG }\end{array}$ \\
\hline
\end{tabular}




\begin{tabular}{|c|c|}
\hline & $\begin{array}{l}\text { TTGCAGANTCAATGCCAAANNACCCTGCTCCGTCCCGCCTAAAAGTCTTCTTCGATGGGGGCT } \\
\text { GCCGCCCNAATCCCCTCTAAGATGGTTACCGCANTCGTTGCGGTCGNAATGCCNACCGTCTTT } \\
\text { CCATATGTCGGCTATGNCCTTNGCTCACAGCANAGTGTCTGTCGCTGATGCCCGC }\end{array}$ \\
\hline $\begin{array}{l}\text { Sinai } 8(\mathrm{R}) \\
\text { Fig } 12\end{array}$ & $\begin{array}{l}\text { NNNNNNNNNNNTNNNNNNCTAGNATGTANTTCTAGGAATNGNNGCCTTCTCGTGTGGTTGCT } \\
\text { AAGGACGCATCGCTGTCTTGTNATTTNNNGNNGGCTGATGGTCNNATCTTTTAGCAACTTCCC } \\
\text { CGCTGCGCCTTTANGNTTCGNCCCAAGTAAGTCTGGCGTTTTAAATGTCTGTTCACCTCGAGG } \\
\text { GAAGCCGGGCTAGACCCAGCCCCGATGCGTTCTGCNACCGCTTAATGTGACGCAGTTCCGTAT } \\
\text { TCTATGCCNAAATCCCGGCTCCCNNCGCGCTCNATCCTCTTCNATTCGTCCCCCCCCTTTCTCG } \\
\text { CTCCCGATGCTTTCCCNATCCGTC }\end{array}$ \\
\hline $\begin{array}{l}\text { Sinai } 22(\mathrm{~F}) \\
\text { Fig } 13\end{array}$ & $\begin{array}{l}\text { NNNNNNNNNNNNNTNATTTNTATANNTNTAAAANAANTCGGTGCGTCTTTCTNCGGTTCTTAC } \\
\text { NAAGNTGACTTGANGCTCAANNGNNGNACGGCTCTNCCATCCCTGCTCGTCAGAAAATTCTN } \\
\text { TGCNNATATGTGGGATTGNGGAGAATCNNTNTCNNCNTNAANCCAANTTCCCCTCGGGCACC } \\
\text { CGGTAANNGGGGTNCNNTAANCTTGGCTCCTCACGNNNCGCCACTGGTGTTGCGCCGCGGGT } \\
\text { GACTCCCTCTATNATTGTCTTAATGACAGTGGGCGCCGCTGCTGGTTNTATTCGTAGGTG }\end{array}$ \\
\hline $\begin{array}{l}\text { Sinai } 22(\mathrm{R}) \\
\text { Fig } 14\end{array}$ & $\begin{array}{l}\text { NNNANNNNANNTTTCTNNNACTGTATANNNTNAGATTCGGTGCGTCTTTTTGCGGNNCNNCN } \\
\text { GANNGTCTAGGGTGCNTCANNTNTAANNGAACGNGCTANNCNAAAAGTTAGATNAAANAAN } \\
\text { TGTTCATTGNGNCGGCNANGTGNAAANGTGNNNNNNNACANCNCNACAGTAAAANNGGNNG } \\
\text { TCCACNGAGATCTAANCGTTGACCGGTGGTNTNNTGTNNTGNCCANTNNNNNGCCACGATTC } \\
\text { CTGCGCGCGNCGGNTCACNTTTTAATACATCTATTAAACTAGGGGGGCGTCACTNACACAATT } \\
\text { TTNNNNGCTCGTTAGCCGCNN }\end{array}$ \\
\hline $\begin{array}{l}\text { Sinai } 26(F) \\
\text { Fig } 15\end{array}$ & $\begin{array}{l}\text { NNNNNNNNNNNGNNNNNNNNNNCNNCCAAANGTNTGNNNNAGCCAGGCCTTCTCCCGTCTG } \\
\text { ATNNGTACNGGGATCGACGNNNTNGNNGGGNNGGNNGNGTGNTNGNAGCACNACNCTCCAG } \\
\text { TGCGCTGCGGCGCNGCGACCACTAATANANCTTGCCCCTTAANCACTGGTCACCTCNNNAGA } \\
\text { NCCGCCCTATACCCANNCCNNTATTATCTGCNACCGTNGATNTATNCAGTNCGNACTCTGNCC } \\
\text { GAATNNTNGGCTCGNTCCNCGNATATATTCNTCGAGGGGTCCC }\end{array}$ \\
\hline $\begin{array}{l}\text { Sinai } 26(\mathrm{R}) \\
\text { Fig } 16\end{array}$ & $\begin{array}{l}\text { NNNNNNCNNNNGNNNNANNGNGNGNNANNNNCGGGACCCCGAGCCGCGCGGCTTTCTTACC } \\
\text { NGGTGATCACCNTNNNNGGCANAGANNTNNTAAGTTAGGNGNCGTGGGGAGCATAGGCGGA } \\
\text { NTCTGNNCAAGCTCGCTTTATNATCAGATCNANCATNNAGCTTGNGGCCTTAAGCACCCGTCC } \\
\text { CCNCGNNTGANCTTNNCTAGGCGCATCCCGTANACGTTCTGCNNACCTCGGATTAGCGCTGTT } \\
\text { GTGTGANAAGTCCCGAGAGANCTTTT }\end{array}$ \\
\hline $\begin{array}{l}\text { Gimmizah } \\
81(\mathrm{~F}) \\
\text { Fig } 17\end{array}$ & $\begin{array}{l}\text { NNNNANNCNNCNGNCNNNGNTGCCGANTCGTCGTGACCGCGATGCGCGAGGCCTTGCCGGTG } \\
\text { TTCGGTCGCCAGATACCGGGCTACGACCATCCCGAGGGTGTGATGACGGGCGTGGAGACNCG } \\
\text { CACCTCCTCTCCGGTGCGCTTCACGCGCGGCGACCACTATCAGAGCTTGAACACTTCAGGTCT } \\
\text { GTTTCCCGCGGGAGAGGGCGCCGGATACCCCGGAGGCATCCTTTCTGCGGCAGTGTGACGGG } \\
\text { ATCGCAGTTGCAGAATCTGTGCGGATGAGCCTGTGCCGTCCCGCGTAAAGGTCTTCTTCGATG } \\
\text { GGGGCTGCCGCCCNAATCCCTCTAATATGGATATCCCACTCCTTGCACTCCGATTGACNACCG } \\
\text { ACCTTCCACATGTCNG }\end{array}$ \\
\hline $\begin{array}{l}\text { Gimmizah } \\
81(\mathrm{R}) \\
\text { Fig } 18\end{array}$ & $\begin{array}{l}\text { NNNNNNNNNNNNCCCTCGNNGNGCGTGANACNANGGGACCGTGCCGCGCGAATGGCNTTAG } \\
\text { CGGCAGCGGCACCATTCGCCGCACCAACAGGTCTGANGNNGGCCGCGTCAGCCAGCATCGCA } \\
\text { AAGCGACTGTTCAAGCTTGCCAACGGACAGCCGTCCACACATGGGTCTTGCGGCGTGAAGCA } \\
\text { GCCGTCACCTCGGGTGAAGCTTGGCTAGGGCGATCCCCGCTAGGTTCTGCGACCGCTTGATGT } \\
\text { AGCGCAGTCGCGGAGTATAGGCCGAAGTCGCAAGCTCGCGGAGGCGCTCGAGACGTTGCGCA } \\
\text { TCGCTCCCGCGCCATTTCACAATCCCGTTTGCTTTCGCGACTACNTCGGCGGCGTCGCCCAAC } \\
\text { ANAATGAACGCAGGCAAGCTAAGTGATTTGGCCACNGTCAGCGCGGGGATCACCGCCAGCCA } \\
\text { CTCCGCNTCCTAGCTGGTGCCATANCGACGTCTGGAAGACGGGCGGCGATCCGACTGCAACG } \\
\text { ACTGCGATGTCCATCGTACCGAGTATTCGGGCCGCCCCGCCATCAAGAGGACCTTTTGCGCGG } \\
\text { GACNGCGANGCGCATCTCCGCTCCTTCTGG }\end{array}$ \\
\hline
\end{tabular}




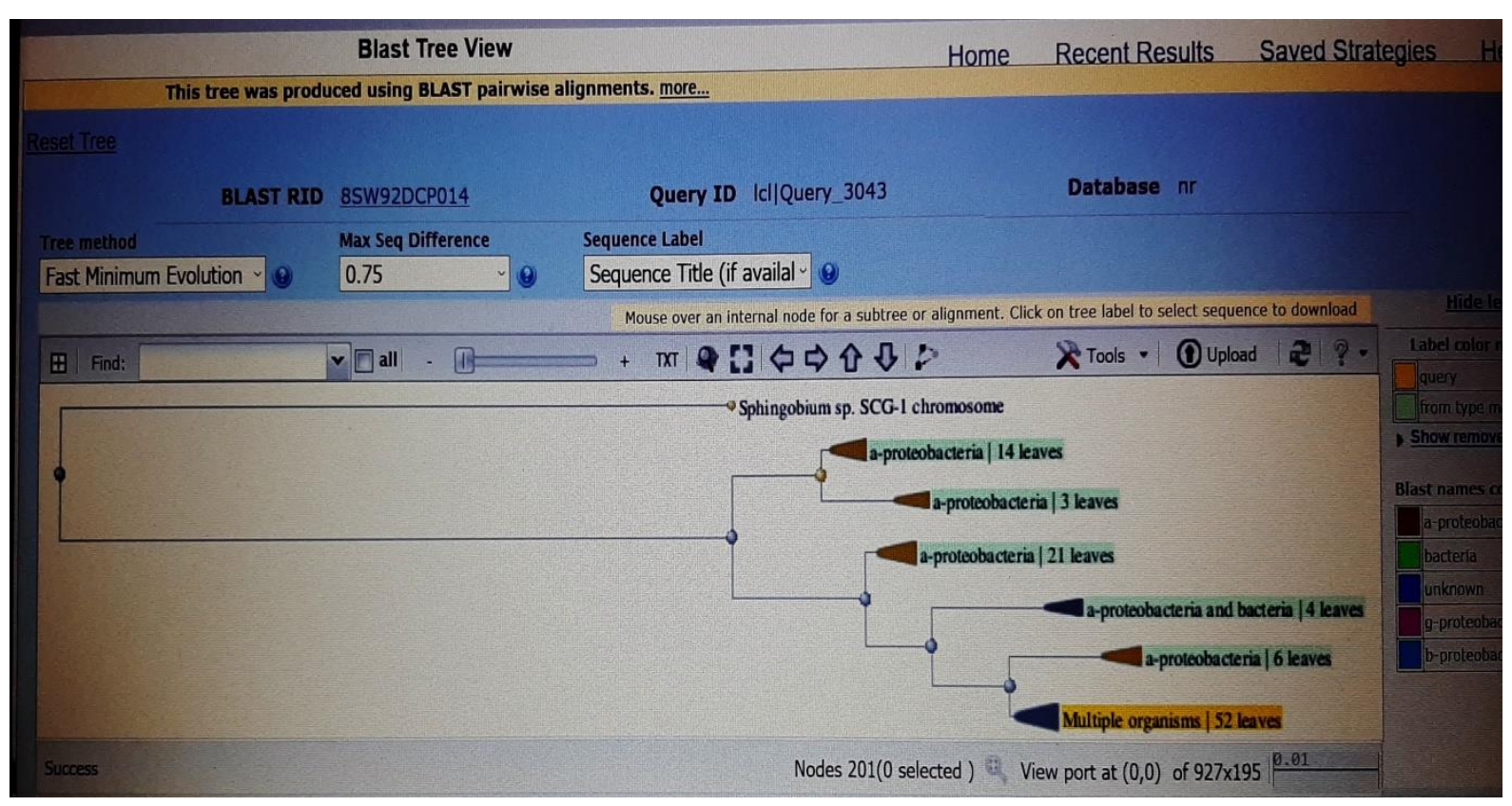

\section{Figure 19: Phylogenetic tree of IGF-1 gene from Gimmizah strain line 81 and other vertebrate species.}

\section{$\underline{\text { 3.4 PCR analysis for TGFß2 }}$}

Transforming growth factor -betas are multifunctional cytokines that have been implicated in the regulation of a broad range of biological processes, including cell proliferation, cell survival and cell differentiation.PCR was used to amplify fragments. The amplified product was $188 \mathrm{bp}$ for lohman strain 7 lines 7,9,12,14,38,58,and 97were appeared band. For Gimmizah strain 3 lines 68,71 and 81 Figure 20 and for Sinai strain 5 lines 6,8,22,26 and 70 were showed sharp band and 7 lines 30,32,35,36,51,64,and 91 were showed firm Figure 21 
M, 1,6,8, $15,22,26,27,28,29,30,31,31$

$188 \mathrm{bp}$

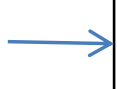

$M, 32,33,35,36,51,57,60,64,66,70,77,91,92$

Figure 21 PCR amplification gel image of TGFß2 gene of chicken (Sinai strain, $M=100 b p)$

\subsection{Bioinformatics analysis of TGFß2 sequence:-}

The sequences of four bands of Lohman line 12,14,58 and 79, three bands of line 8,22 and 26 and one band of line Gimmiah line 81 were purified, sequenced figures from 22to 35 and alignment using BLASTx and BLASTn search service at NCBI website.The sequences from eight line for the three strains were aligned and compared with that of other different representative species using Blastx and Blastn.The dendogram tree was produced using BLAST Pairwise and was 
cleared two branches.The tree was found the relationship of TGF $\beta 2$ for Lohman strain line 14 with Methylobacterium sp. And Mesorhizobium sp. The TGF $\beta 2$ for Lohman strain line 14 is related to Methylobacterium sp.(Fig36) For strain Lohman line 14 showed aligned with reverse transcriptase- like protein in( Sphingomonas sp.), ribonucleae HI, hypothetical protein B7Y4904040 and hypothetical protein ASF0008485.Line 58 in Lohman strain showed alignment with reverse transcriptase- like protein and ribonuclease $\mathrm{H}$ family protein.Line 8 in strain Sinai showed alignment with hypothetical protein in (Curtobacterium)and single transduction histidine kinase.Line 6 in Sinai strain showed alignment with hypothetical protein 11ap6319 (Limosa lapponicabaueri), hypothetical protein N33809572 (Podiceps cristatus), hypothetical protein N32308862 (Cathartes aura), hypothetical protein N30605151 (Opisthoco hoain) and reverse hypothetical protein (Limos sp.). For strain Sinai line 22 showed alignment with NAD(p) FAD-independent oxioreductase in Porphyro bacters wp054117228.1 and hypothetical protein B7Y47 14385 (Sphingomonas sp.) BK 235.For strain Gimmiah line 81 showed alignment with hypothetical protein RLOC (Lonchura striate). hypothetical protein N300 (Calypte anna), hypothetical protein Du 187 (Hirundorustica rustica), hypothetical proteinA52802364 (Pygoscelis adeliae) hypothetical proteinN30003399 (Calypte anna) and hypothetical protein N 32608555 (Eurypyga helias).The dendrogram was shown two branches the first branch included two clusters of Gallus gallus BAC clone and the second branch was shown 6 clusters of birds, hawks and eagles. (Figure 37)

\begin{tabular}{|c|c|}
\hline $\begin{array}{l}\text { TGF } \beta 2 \\
188 \mathrm{bp}\end{array}$ & \\
\hline $\begin{array}{l}\text { Lohman } \\
14(\mathrm{~F}) \\
\text { Fig } 22\end{array}$ & $\begin{array}{l}\text { GNNNNNNNNNNNNNNNNNNNNNGNNNNNGTGNGGNNNACGAGGGGACCTGGCCGCGCGAATGG } \\
\text { CAGCGGCACGATTCGCCGCANNNANNGGNNNNCACGTGGCCGCGTCGCCAGCATCGCAAAGCCA } \\
\text { CTTGCCAACGGACAGCCGTCCACACATGGGTCTTGCGGCGTGAAGCAGCCGTCACCTCGGGTGAA } \\
\text { GGGCGATCCCCGCTAGGTTCTGCGACCGCTTGATGTAGCGCAGTCGCGGAGTATAGGCCGAAGTC } \\
\text { GCGGAGGCGCTCGAGACGTTGCGCAAGGGCTCCCGCGGCATTTCACAATCCCGTTGCTTTCNCGA } \\
\text { GCGGCGTCGCCCAGCAGAATGACGCAGGCAANCNAAGTGATTTGGCCACGGTCGGCGCGTGGAT } \\
\text { GCCACTCCGCGTCCGAGCTGGTGCCATAGCCGACGTCTGGAAGGACTGTCGGGAATCCGGGTGCA } \\
\text { CTCCTCTCTTTCTTAGCGGGAGTCGGGGGGGGAAAACCAAGATAGGGAAGAAGACCGTTGGGNNG } \\
\text { ATGGTCATCGCCGATGTTTCTGCGGCCTTGGTGCGGGCCACTGCGGGAGAGAGGAGGCCCCCGGA7 } \\
\text { GCCCTCTCCCGGG }\end{array}$ \\
\hline $\begin{array}{l}\text { Lohman } \\
14(\mathrm{R}) \\
\text { Fig } 23\end{array}$ & $\begin{array}{l}\text { ANNNNNNNNNNNNNCNNNNNNNNNNNNNNNNGCNGNNNNNNNNNCNCCNNANGCGCGATGTG } \\
\text { TGGGGGGCAGGGCNCATTCCCGGACCNCCNNNNAGCGNTGGNNGCGNGGCCTGCAGAGCAACA } \\
\text { CNACTGTTCANGCTTGCTAAACGACCNGCGACCACACNTGGATCTTGCGGCCTTAAGCAGCGGTT } \\
\text { CCCTCGAGAGAAGCTTGCCTAGACCGATCCCCGCTAGGTCTGCNACCGCTTGATGTGACGCAGT } \\
\text { CCCGGANTATAGGCCGAAGAGCGAGGTCGCGGNGGCGCTCGGATACTTGCGCATCGCTGCCGC } \\
\text { GCCCTTGCACACTCCCGTATGAATTCNCGACTACTTCCGCTCCGTCNGCCGACAGAGTAACCNCA } \\
\text { TGGCAGCTATGCATCTGGCCACACTCGGGCGGGGGTCANTGCTGGCCGAGCCGATCCCAAGCTG } \\
\text { GATGGCTTAGCCTTACGGCTCGGGAGATGCGGTCTGGGGGTGCGGGGTGGGACGTGTGN }\end{array}$ \\
\hline $\begin{array}{l}\text { Lohman } \\
58(\mathrm{~F})\end{array}$ & $\begin{array}{l}\text { GNNNNNNANNNNNAGNNGANNNNNGTGGNGNAGTTGGCCGTGGCCCTATGGCTGAAGGCTGGG } \\
\text { AACAGGANNNGAGGGGGCCTCCCCACCTTGTGCCTGCTGGCGGTTGGGGGGAGGTGGAGAGAG }\end{array}$ \\
\hline
\end{tabular}




\begin{tabular}{|c|c|}
\hline Fig 24 & $\begin{array}{l}\text { TCAGGGGGGCTCCCGAGGCTACTCTCCAANAAGGTCCTGGTGTTGGTGGGCGGATGGNTTGGTT } \\
\text { GNCCGAGGAGATCCGGCGAGGTTCTGCGACCGCTTGATGTAGCGCAGTCGCGGAGTATAGGCCG } \\
\text { AAGTCGCAAGCTCGCGGAGGCGCTCGAGACGTTGCGCATCGCTCCCGCGGCATTTCACAATCCC } \\
\text { GTTTGCTTTCGCGACTACNTCGGCGGCGTCGCCCAGCAGAATGAACGCGGGCAAGCTAAGTGAT } \\
\text { TTGGCCACGGTCAGCGCGTGGATCANCNCCAGCCACTCCGCGTCCGAGCTGGTGCCATAGCCGA } \\
\text { CATCTGGAAGGACGGTCGTCAATCCGAGTGCNACNACTGCNATCTCCATCTTACCNNNATTCGG } \\
\text { GCNGCACCCCCATCGAAGAAGACCTTTACCNNTACGGCACGNACTCATCTCCACTGCTTCTGCGC } \\
\text { CTTGATCCCTCCGCTGCNNAGAAGAGATGGCGCCGG }\end{array}$ \\
\hline $\begin{array}{l}\text { Lohman } \\
58(\mathrm{R}) \\
\text { Fig } 25\end{array}$ & $\begin{array}{l}\text { NNNNNNNNNNCTGCTNNNGNGNTGNGGTGAGGTGGNGNTGGCCTCTTCGCCAGTAACAAGCGA } \\
\text { GGGAGGAGGGGANGCCCCNGGTGGGGGAGGGGGGGTCCAGGGAGGATNANGGNATNANTGCG } \\
\text { CTGAAAGGGCGAGGACGANNATNGAGCTTGAACCCTTCATGTCTTTTTATTCTCGGGAGAGGNC } \\
\text { GCCCGATACCCCNGANGCATCCTGTCGGGGGGCAGTGTACGGGATCGCAGTTGCAGAATCTNTG } \\
\text { GCTATGAGCCTGTGCCGTCCCGCGTAAAGGNNCTCTTCGATTGGGGGCTGCCGCCCTAATCCCTC } \\
\text { TAAGATGGTTATCNCAGTCCTTGCGCTCGGATTCACNACCNTGCTTCCACATGTCGGCTATNNCA } \\
\text { CCAGCTCNGACACGAAGTGNGTNCACTGATCCACNCTCTTTTTTTTTCCGATCACTTCNCTTACC } \\
\text { CTCGTTC }\end{array}$ \\
\hline & $\begin{array}{l}\text { GNNNNNNNNNNNNNGANCTNCCTGGNGCACTTGNNTCCCCCCATCTCTGATTACTGTGAGCGGG } \\
\text { GNNNGAGCGCCTCCCCCTCTTCTCCCTTCTCTCTTTTTTAGGGAGTTGTAAAGAGTCATGTGGGCC } \\
\text { CTCCCTCCTATCCTCTAATAAGGTAACTAGTGTAGGTGAGAAGGATGGCTTTAAGTTGCTCCAGA } \\
\text { GGAGATTCAGGTTGGTATTTGGCTACAACCGCTCCAGGTGACGCAGCCCCGTATTCTGNGCGTAT } \\
\text { TGGCGAGTCGTCCCGCTCCCTGTCCTCGTCTTTTCGCTCTGCCGCCCTTTCCCCTCTCCTATGATTT } \\
\text { CCNACTACTTNGGTCNGTTTCCCACATTGCTTTCNNCTTAANTTAATCANTATCTCTTATA }\end{array}$ \\
\hline & $\begin{array}{l}\text { CNNNNNNNNANCTNGCTGNNNNNNGCTGTNGTGAGGTGNNNTTGGCCTCTTCTCCCAGGTAACA } \\
\text { GTCATAGGAATGAGGNANANCCTCCGTTGGGGAGGGGAGGATGCTGGGTGTATTTATACTCTAA } \\
\text { ATNCTNGAAATACACANGCNCAACACATNAGCTTCCCGCATTTAACACTGGTTGCCTCGAGTGA } \\
\text { GCCGAGCCTNACTCCCCCTCGTTTTATGTGCATCTCATTGCTCCCGGTGATCGATGCCCCGTATTC } \\
\text { AGCCGCCNATAAGCACGAGCATGGTGAGCCGCCGTCTACCGCGCCATTCGCCGAGGCCGTCCTC } \\
\text { GCCACCTCTCCTATGATTACCCNACTCATTCGGGCCNGTTGGCAACCGGGCTTCCGTTTGCGATT } \\
\text { GTTGCAAGACCCACCCAACTTCTCTTGACTCNAGCAGACCCCCGCTATGCCCTAACTGTTCCCTT } \\
\text { ACCCGACCGCTCGTACCTGCCTCCAGGGAGATGCGGAGAATGTCT }\end{array}$ \\
\hline & $\begin{array}{l}\text { NNNNNNANNNANNNNNANCTNNCTGGAGCACTTACCATTTCCCTNTCTCTGAATCATGTTTACAT } \\
\text { GAGAAATGACGGCCTCCCCACCTTTACCCTACTTCCTTTTTCAGGGAGGTATAAAGAAACATAGT } \\
\text { GTCTCCCCAGCCTACTCTTCTATAAGGAAACTAGTGATAGGGTGAGAAGGAATGGCTTTAAGTTG } \\
\text { CTCCAGAGGAGATTCAGGTTGGATATTAGGAAAAT }\end{array}$ \\
\hline & $\begin{array}{l}\text { NNNNNNNNNNTGCTNANNAGTGTNGTGANNNNNGGTTGGCTNNTCNCCCAGGTAACTAGCAGA } \\
\text { GGGATGATGNNANGNNCCANTTGCTNNGTGAATTCCAGGTTGTATAANTGAAAAACCCTCAAAA } \\
\text { TACGCANGANCACAANANNCNNGTNTCGGGTGTTACCTACCGAGATGCTTGTTAATTNATCNGN } \\
\text { TACTTTCTCCACACTTACTGTGA }\end{array}$ \\
\hline & $\begin{array}{l}\text { NNNNNNNNNANNNANCTNGNNCTNNNGTGGNGCAGTTGANTCGCCCNNTCTCCGATTGCTGGG } \\
\text { GAAAGGAGAAAAAGGGGANCNCCCCCCCTGTGCCTTCTTGCGGTTGGGGGGAGGTANAGAGAG } \\
\text { TANTGAGGGCTCCGAAGCACCCCCTATAATAAGGTNNATCTTGCAGGTTGAGCAGGGGTGCCCT } \\
\text { CGGGTGAGGCTGGCGGAGACCCCGCCAGGCTAGGTTCTGCGACCGTTTGATGGACACAGTCCCA } \\
\text { GAATCTANGCCTATAGGCCGGTTCGTCCCGCGTAAAGATCTTCNTCGATTGGATCCCGCCGCCCT } \\
\text { TACCCCATCTCATATGAATATCCGACTCGTTGCGCTCCAATTTCCCAACNNACCTTCCACATNCA } \\
\text { GGCTATNNCAGCAGCTCGCTGCCNNAGTGTNTGATCNCTGCTGCCCGCTCCTGATCCTAGNTGGA } \\
\text { TCACTTAGACTGTGCTCACGTTCATGCTGCTGGGAGACGGGGGACGACGTAGTCGCGTGAACGT } \\
\text { CA }\end{array}$ \\
\hline & $\begin{array}{l}\text { CNNNNNNNNNTGCTGNNNNNNNTGNGGTGAGGTGNNNTTGGCCTCTTCTCCCAGGTAACAGNCA } \\
\text { TAAGGATGANGGAANGNCTCNGTTGGCGAGGGGGGGGTGCTGGGTGGATATTAGGCTCNATTTC } \\
\text { TGAAGATGCGGAGNNGAGCACANANCTTGGNTCTTGCGGCTTAAGCAGCCGTCACCTCGNGTGA } \\
\text { GCTTGCCTAGGGCNATCCCCCTAGGTTCTGCNACCGCTTGATGTGACGCAGTCCCGTATATATGC } \\
\text { CNAATTGGCGGGCTCGCGGAGGCGCTCGGGACGTGGCNCTTCGCTCCCGCNCCATTTCCCATCCC } \\
\text { TATTGCTTTCCGACTACGTCGGCGNCGTCGCCCAGCAGAATATACGGGGGGCGAACTATGTCGAT } \\
\text { TTGGCCACTGTCAGCGCTTGGGATCAACGCCGNCCACTCCGCGTCCNAGCTGGTGCCATAGCCGA } \\
\text { CGTCTTGAANAGTCAGGCGGGGATCCGGGGGCGACGTCTTGGGA }\end{array}$ \\
\hline $\begin{array}{l}\text { Sinai } 22 \\
\text { (F) } \\
\text { Fig } 32\end{array}$ & $\begin{array}{l}\text { NNNNNNNANNAGCNGNNCTNNNGNGGNGCANTTGNTGTGGCCCTATCGCTGATGACTGGGAGA } \\
\text { GGGGGANANGACGGCCTCCCCACATCCTGCCTTCTGGCGGTTGGAGGGAGGGGAAAGGAGTAA } \\
\text { GGTGGGCCCTGAGGGCTATCCTCTAATAAGGTGCTAGTGTAGGTGAGAAGGATGGCTGTAAGTT } \\
\text { GCTCCAGAGGAGANNAGGCAGCCTTTCTGCGACAGTGNACTGTATCAAGGTTGCAGAATCTGTG } \\
\text { CCGATGAGCCTGTGCCGTCCCGCGTAAAGGTCTTCTTCGCTGGGCTCTCCCGCCCTAATCCCTGT }\end{array}$ \\
\hline
\end{tabular}




\begin{tabular}{|c|c|}
\hline & $\begin{array}{l}\text { AATATGCATATCCCACTCCTTGCACTCNGATTGACAACCGTCCTTCCACATGTCAGCTATGGCAC } \\
\text { CAGCCCGGACGCGGAGTGGCTGGCGCTGATCCACGCTCTGACCGTGGCCAAATCACTTCNCTTGC } \\
\text { CCTCGTTCATTCTGCTGGGCGACNCCNCCAACGTATTCGCGAAANCTAACAGGATTATTAGATGC } \\
\text { CGCGGCGACNATGCACAACATCTCTANCGCCTCCNCGAGCTTGCNACTTCNGTCTATACTCCGCG } \\
\text { ACTGCNCTACATCATACNGCCGCAAAACCTCNNNGGATCGCCCCTANCCCACCTTCACCCAAGG } \\
\text { TGAANNCTGACTTCACACCNTATACTCATAGTTTGAACGGCTNTCGNTTAG }\end{array}$ \\
\hline & $\begin{array}{l}\text { NNNNNNNNNNNNNGCTNANGAGTGNGGTGAGGNGGGGTCGGCCTCTTCTCCCAGGTAACAGNG } \\
\text { ATAGGNAGGGNGGGNNGGNTNNGTGGGGGGGGGGGGGATGTTGGGAGCATAGTATTCTCAGAA } \\
\text { ATAGCGGAGAGGCGCAGGGCCGCACATAGATCTTGCGGCGTGAAGCTCTCTTCCCCTCGGGTGA } \\
\text { AGCTTGCCTAGGCCCATCCCCNCTAGGTTCGGCGACCGCTTGATGTANCGCAGTCGCGGAATATA } \\
\text { GGCCNATGTCGCTGCTCGCGGAGGCGCTCGATACTCTTCGCTNCGCTCCCGCGGCATTTCACNCT } \\
\text { CCCGTATGCTTTCNCGACTACTTCCGCTGCGTCGCCCAACAGAATTACCACATGCAAGCTATGTG } \\
\text { ATTTGCCCACAGTCAGCGCGTGTATCACTGCCCNCCACTCTGCNNNCGANCTGGTGCCNNANNC } \\
\text { GACTCTCGTTGGACTGCTTCGATACGACTCCAACGACTGCGATATCCCTCTTACCATTATTCNATG } \\
\text { CGCGNCCNCATATGANCAANACCTGNAGCGCCTNCNCGAGNTCTCGACTCCAGTGCTTCTGCAC } \\
\text { CTTTGTGCCATCCNNGCCGAAGAGGGACTCCTCCGGGATCTCNNGGCCCTCGCTCNCNGAAAGA } \\
\text { AGCTGACTTGTAANGCTCTAATAGTGTGCAGCAGGCTTAAGCCGCGGCGGATAGGAGGTGCGCG } \\
\text { TCTGCAGAGCCCGGGGGTCAC }\end{array}$ \\
\hline & $\begin{array}{l}\text { GNNNNNNNNNAGNNGTNCACTTGTGNAGTNGTAGGCCATGCCCNCNTNTCNGATTAGCTGTTAN } \\
\text { CATGAGAAAAGGCCGACCCCCCACCTTGTCCCATCTTCCTTTTAGGCAGAGGTATAGAGCAATAA } \\
\text { GGTCTCTCCTGAGCCTACTCTTCTATAAGGTAACTAGTGTAGGTGAGAAGGAATGGCTTTAAGTT } \\
\text { GCTCCAGAGGAGATTCAGGTTGGTTATTAGGAAAAATCACTTTGACGCTATCCCGTATTATAACC } \\
\text { CNAAATGACGTGTCGCTCCAGGCGAACAATACACGTCGTTACGCTCCCACGCCCTTAACCAACCC } \\
\text { CATATTAATTACNCNACTACTTCNACTCANTCTCCCAACANATTTTACTTATATAAACTAAGTCA } \\
\text { C }\end{array}$ \\
\hline & $\begin{array}{l}\text { NNNNNNNNNNNTGCTNNNNNAGCTGTGGTGAGGTGNNGTTGGCCTCCTCTCCCAGGTAACAGGC } \\
\text { ANAAGGATNNNGGAANGCCNCCAGGTTGCACAAGTGGAGGTTCAGGGAAGATACTATGCTCAA } \\
\text { AAATACAGANGATGCGCAGAGGGCACACANTCTATCTTGCCGCATTAAGCAGGCGTCACCTCGA } \\
\text { GTGAGCCTACCCTAACTCCATCCCCTCTATGTTCGTCTNCCTGCTCAAGGTGATCGCTGTCCCTTA } \\
\text { TTCTAACCCCAATAAGGCCGGCTCGCTTAGCCGCTCNATCTGCGTCGCGTCNCTCCCTCGCCACT } \\
\text { TCCCCTTCCCATATGATTACNNGACTACTTCGGTCCTCTCCCCNNNATGTTCTCACTTCCNGCTAT } \\
\text { TCAATTATTTCACATACAACGGCTAAATAAAAGAAACCACGCCCCTTCCAAGCTGGTCCCTAAGA } \\
\text { TGACGTTTGGAGAAAAGTTACNNGATCCNGGGCCAGCGTC }\end{array}$ \\
\hline
\end{tabular}

Figures from 22 to 35 showed the sequences of each line for all strains used in this study of TGFß2 gene

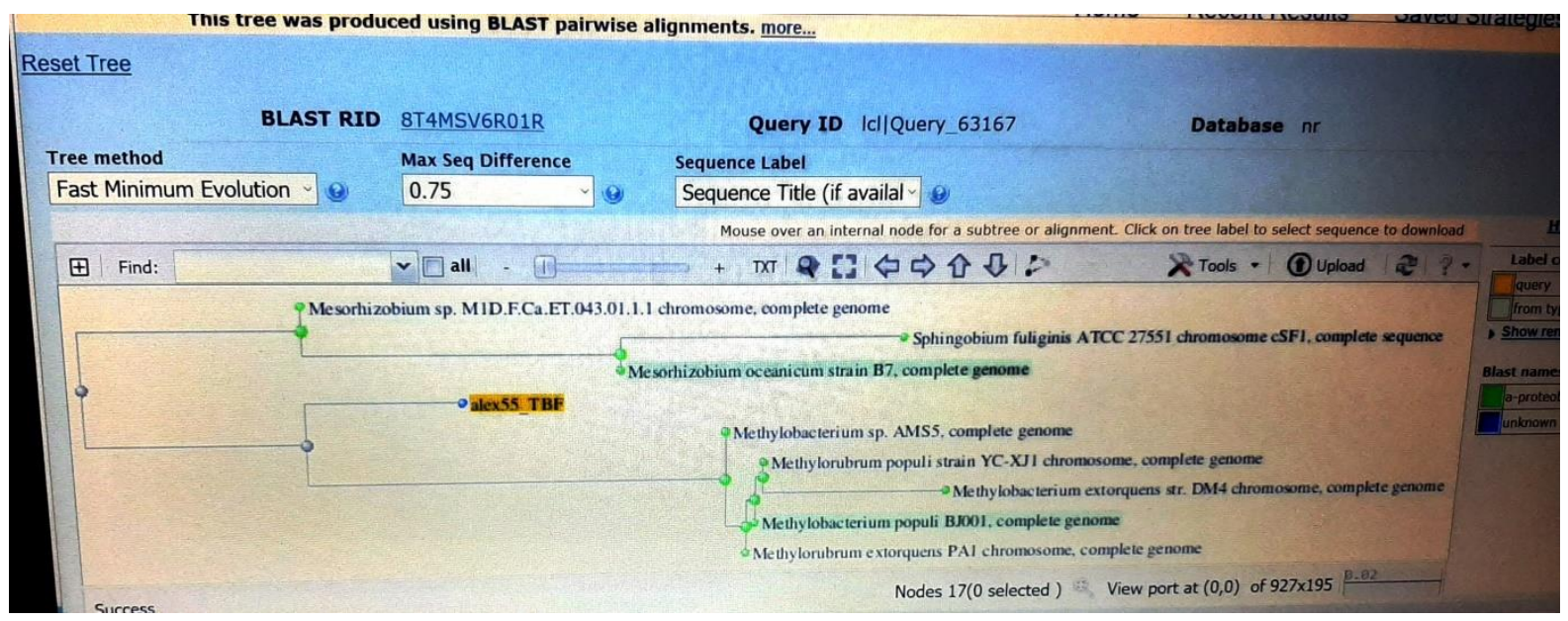

Figure 36: Phylogenetic tree of TGFß2 gene from Lohman strain line 14 and other vertebrate species. 


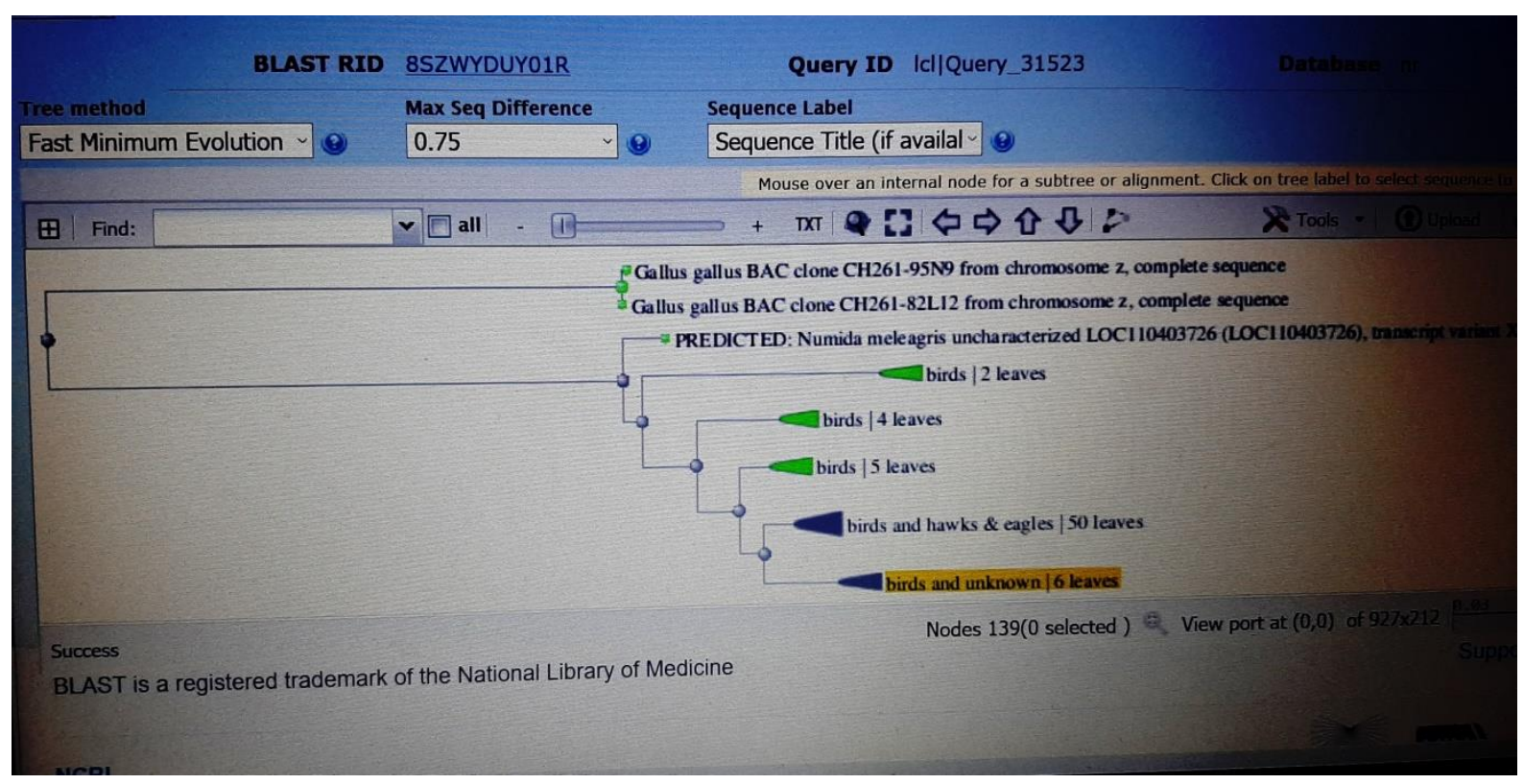

Figure 37: Phylogenetic tree of TGF 2 gene from Gimmizah strain line 81 and other vertebrate species.

\section{Discussion:-}

\section{$\underline{4.1 \text { IGF-1 gene }}$}

The amplified product was 813 bp that the amplicon was IGF-1 This has been shown to be the case in different studies that showed the same size for IGF-1 (Zhou et al., 2005)The study of applicant genes is one of the primary methods to determine whether specific genes are related to economic traits in farm animals (Zhou et al., 2005) It is known that the genes of the growth axis play crucial roles in the regulation of growth, development and differentiation. The genes of the growth axis probably affect the meat quality traits of animals (Lei et al., 2007).An alignment with the deduced amino acid sequence revealed a high degree of homology to chicken in hypothetical protein, NAD(P) FAD dependent oxireductase in lohman, Reverse transcriptase - like protein and hypothetical protein in Sinai and Gimmizah. The highest homology observes was $100 \%$ with sphingomonas sp. in Sinai strain (line 8),93\% in Lohman strain (line 97) with methanoica, 69\% in lohman strain(line 97) with Sphingomonas sp. , 75\% in Gimmizah (line 81) with Sphingomonas sp. And 78\% in Gimmizah (line 81) strain with Caulobacter $s p$. The results showed that IGF-1 was appeared high sequence identity with other species.IGF-1 exerts a general effect on overall body growth and expressed in both liver and muscle tissues such as muscle may be the predominant source of IGF-1 during prenatal chicken development (Pandey et al., 2013) 


\subsection{TGFß2 gene}

TGF $\beta 2$ regulates a number of biological activities related to cell proliferation and differentiation, embryonic development, myogenesis and osteogenesis . In chicken, Bennett et al ., (2007)revealed significant association of TGF $\beta 2$ with body weight and explained its role in bone resorption and remodeling. TGF- $\beta \mathrm{s}$ have bi- functional roles in the immune system, whereby they regulate both proinflammatory and anti- inflammatory activities( Okamura et al., 2015).An alignment with the deduced amino acid sequence revealed a high degree of homology to chicken in hypothetical protein in Lohman and Sinai stains, reverse transcriptase- like protein in Sinai, Gimmizah and Lohman strains. The highest homology observes was $100 \%$ with Paenarthrobacter aurescens , $90 \%$ in Methylorubrum extorquens , 76\% in Sphingomonas paucimobilis in Lohman strain( line 14).In Gimmizah (line 81) was shown homology with $85 \%$ in Gallus gallus, $83 \%$ in Aquila chrysaetoes and $82 \%$ in chicken DNA sequence. While high homology was appeared in Sinai strain (line 6) with 86\% in Aquila chrysaetoes , 91\% in Gallus gallus Bac clone and 93\% in Gallus gallus uncharacterized.The TGF- $\beta 3$ gene was implicated in having a role in determining growth and feeding traits in current yellow meat- type chicken population (Jin et al., 2013). The chicken genome has a haploid content of 1.2 $\mathrm{X} 10^{9}$ of DNA approximately $40 \%$ that of either mouse or human. The latest version of the chicken genome database chick GBASE is part of the comparative mapping database for farm and other animals .The TGF- $\beta 2$ is a multifunctional molecule that can stimulate or inhibit cell growth according to the cell types and the presence of other growth factors.In chicken, during early development the expression of TGF- $\beta 3$ has a role in regulation of growth, an association of TGF$\beta$ with body composition in chickens (Pandey et al., 2013) The TGF- $\beta 2$ plays an essential role in the development of a wide range of tissues and organs.

\section{Conclusion:-}

The study was able to assess polymorphisms on the IGF-1 gene as the candidate gene with increased meat production in chickens in Egypt.IGF-1 confirming high sequence identity among species which suggest an important role of these growth factors conserved during evolution.

\section{Acknowledgments}

Research supported by The Science Technology Development Fund (STDF, 2418). 


\section{All authors has no conflict of interest}

\section{References:}

Akaboot, P., Duangjinda, M., Phasuk, Y., \& Kaenchan, C. (2012). Genetic characterization of Red Junglefowl (Gallus gallus), Thai indigenous chicken (Gallus domesticus), and two commercial lines using selective functional genes compared to microsatellite markers, 11(3), $1881-1890$.

Allistone E, Piek E, Derink R 2008 TGF- $\beta$ family signaling in skeletal development maintenance, and disease In: Derink R, Miyazono K, editors. The TGF- $\beta$ family. Cold Springer Harbor: Cold Springer Harbor Laboratory Press. P 667- 723.

Amills M., Jimenez N., Villalba D., Tor M., Molina E., Cubilo D., Marcos C., Francesch A. Sanchez A., and Estany J. 2003 Identification of three single nucleotide polymorphisms in the chicken insulin- like growth factor 1 and 2 genes and their associations with growth and feeding traits. Poult. Sci. 82, 1485-1493.

Beccavin, C., Chevalier, B., Cogburn, LA.,Simon, J., Duclos, M.J., 2001 Insulin- like growth factors and body growth in chickens divergently selected for high or low growth rate. J. Endocrinol.168 : 297-306.

Bennett, A.K.,Hester, P.Y., Spurlock, D.M.,2007 Relationships of transforming growth factor $-\beta 2$ single nucleotide polymorphismand messenger ribonucleic acid abundance with bone and production traits in chickens. Poult.Sci. 86, 829-834.

Burt, D.W. and I.R. Paton. 1991. Molcular cloning and primary structure of the chicken transforming growth factor - beta 2 gene DNA Cell Biol. 10: 723-734.

Burt, D.W., and A.S. Law. 1994. Evolution of the transforming growth factor - beta superfamily. Prog. Growth factor Res.5: 99-118.

Buyse, J., Decuypere , E., 1999. The role of the somatotrophic axis in the metabolism of the chicken. Domest. Anim. Endocrinol. 17, 245-255.

Conlon, M.A., Kita, K., 2002 Muscle protein synthesis rate is altered in response to a single injection of insulin- like growth factor- 1 in seven day-old Leghorn chicks. Poult. Sci.81, 1543-1547.

Dabovic B, Rifkin DB. 2008. TGF- $\beta$ Bioavailability: Latency, targeting, and activation. In: Derink R ,Miyazono K,editors. The TGF $-\beta$ Family. Cold Springer Harbor: Cold Springer Harbor Laboratory Press. P 179-202

Fang, L.H., W. Zhu, K. Chen, X.Wu, Q. Tang and Y. Gao 2008 Associations between GHR and IGF-1 gene polymorphisms and reproductive traits in wenchang chickens. Turk. J.Vet. Anim. Sci., 32:281-285

Florini, JR., D.Z.Ewton and S.A. Coolilican,1996 . Growth hormone and the isulin-like growth factor system in myogenesis. Endocr. Rev., 17: 481-517. 
Jakowlew, S.B., Dillard, P.J., Sporn, M.B., Roberts, A.B., 1988. Complementary deoxyribonucleic acid cloning of a messenger ribonucleic acid encoding transforming growth factor factor beta 4 from chicken embryo chondrocytes. Mol. Endocrinol. 2 (12), 1186-1195.

Halper, J., urt, D.W., Romanov, M.N., 2004 On reassessment of the chicken TGFB4gene as TGFB1. Growth factor . 22(2), 121-122.

Heine U, Munoz EF, Flanders KC, Roberts AB, Sporn MB.1990 Colocalization of TGF-beta1 and collagen I andIII, fibronection and glycosaminoglycans during lung branching morphogenesis Development 109: 29-36.

Jin S., Chen S., Li H., Lu Y., Zhang D., Ji C., Xu G., and Yang N. 2013 Polymorphism in the transforming growth factor $\beta 3$ gene and their associations with feed efficiency in chickens. Poultry Science 1745-1749.

Kingsley DM. 1994 .The TGF- $\beta$ superfamily: new members, new receptors, and new genetic tests of function in different organism. Genes Dev 8: 133-146.

Lee P. D., Peacock A., Roessler M.K., Hester J., and Reeves J.T. 1989 Insulin- like growth factor (IGF)-I and IGF- binding activity in normal and fast- growing chickens. Life Sci . 45, 2465-2470.

Lei M., Luo C., Peng X., Fang M., Nie Q., Zhang D., Yang G., and Zhang X. 2007 Polymorphism of growth- correlated gene associated with fatness and muscle fiber traits in chicken. Poult. Sci.86, 835-842.

LeRoith, D., 1996 Insulin- like growth factor receptors and binding proteins . Bailliere's Clin. Endocrinol. Metab.10, 49- 73.

Li H. F., Zhu W.Q., Chen K.W., Wu X., Tang Q.P., and Gao Y.S. 2008 Association between GHR and IGF-I gene polymorphism and their reproductive traits in wenchang chickens. Turk. J. Vet. Anim. Sci. 32, 281-285

Mahmoud, T.H., Sayed I.F., Madkour Y.H., and Honein M.M. 1982 The Gimmizah a new breed of chckens. Agri Res. Rev., 60:51-67.

Moriyama, S., Ayson, F.G., Kawauchi, H., 2000 Growth regulation by Insulin- like growth factor -1 in fish . Biosci. Biotechnol. Biochem. 64, 1553-1562

Ohlsson, C., Mohan, S., Sjogren , K., Tivesten, A., Isgaard, J., Isaksson, O., Jansson, J.O.,Svensson, J., 2009 The role of liver- derived insulin- like growth factor-I. Endocr. Rev. 30, 494-535.

Okamura T., Morita K., Iwasaki Y., Inoue M., Komai T., Fujio K., Yamamoto K. 2015 Role of TGF- $\beta 3$ in the regulation of immune responses.Cline Exp Rheumatol 33 s63-s69.

Pandey N., Singh R.P., Saxena V.K., Shit N., Singh R., Sharma R.K. 2013 Effect of IGF-1 gene polymorphism and expression levels on growth factors in Indian colored broilers. Lvestock science 155: 157-164.

Pardali, E., TenDijke, P., 2012 TGF beta signailing and caediovascular diseases .Int. J. Biol.Sci.8, 195- 213 
Patel R.K. and Jain M. 2012 NGS QC Toolkit: A Toolkit for quality control of next generation sequencing Data. PloS One 7, e30619.

Piek, E., C.H. Heldin, and P.Ten Dijke. 1999. Specificity, diversity, and regulation in TGFbeta superfamily signaling.FASEB J. 13: 2105-2124.

Roberts, A.B., and M.B.Sporn. 2012. Transforming growth factor - eta: A large family of multifunctional regulatory proteins. J. Anim. Sci. 66: 67-75.

Ruffier M., Kahari M., Komorowska M.,Keenan S., Laird M., LongdenI., Proctor G.,Searle S., Staines D., Taylor K., Vullo A., Yates A., Zerbino D., Flicek P. 2017 Ensembl core software resources: strong and programmatic access for DNA sequence and genome annotation. Database Oxford bax020.

Sakar M.A., Gado, M.S., and Elokil A.A., 2014 Molecular isolation , cloning and sequencing of chicken Insulin- like growth factor 1 ( IGF-1) gene from high producing exotic breed to produce transgenic chickens of native breeds in Egypt . Animal Biotechnology (Poultry and fish), 1-6.

SAS, 2004 Institute. SAS, user's Guide: statistics SAS, Institute Inc. Cary, NS, USA..

Saxena V.K., Sachdev A.K., Gopal R. and Pramod A.B. 2009 Roles important candidate genes on broiler meat quality. World's Poultry Science Journal 65: 37-50.

Serra R, Pelton RW, Moses HL. 1994 TGF $\beta 1$ inhibits branching morphogenesis and N-myc expression in lung bud organ cultures development 120: 2153-2161.

Sewalem A., Morrice D.M., Windsor D., Haley C.S., Ikeobi C.O.N., Burt D.W. \& Hocking P.M. (2002) Mapping of quantitative trait loci (QTL) for body weight at 3, 6 and 9 weeks of age in a broiler layer cross. Poultry Science 81: 1775-1781.

Zapf, J.and E.R. Froesch. 1996. Insulin- like growth factor-1 actions on somatic growth. In Handbook of physiology. J. L. Kostyo, ed. Oxford university Press, New York.

Zhou H, Mitchell AD, McMurtry JP, Ashwell CM, Lamont SJ (2005) Insulin- like growth factor I Gene polymorphism associations with growth, body composition, skeleton integrity, and metabolic traits in chicken Poultry Sci. 84: 212-219. 